\title{
AN APPLICATION OF A SWITCHING REGIMES REGRESSION TO THE STUDY OF URBAN STRUCTURE*
}

\author{
by \\ Gershon Alperovich \\ and \\ Joseph Deutsch \\ Department of Economics \\ Bar-Ilan University \\ 52900 Ramat-Gan \\ Israel
}

Revised January 2001

* The authors are grateful to anonymous referees for their constructive comments that helped to improve the exposition and content of the paper. 


\section{Introduction}

Analyses of urban structures are traditionally conducted using population and employment density functions, which describe the behavior of these densities as a function of the distance from the Central Business District (hereafter - CBD). The three most critical assumptions underlying this approach posit that the urban area is monocentric, land around the CBD is perfectly uniform in all directions and at all distances and housing capital is perfectly malleable. The assumptions are well rooted in a large body of theoretical modeling, its origin dated back to the seminal works on urban structure by Muth [1969] and Mills [1979]. These authors established that introducing the location choice of economic units in spatial models by using only one variable - the distance from the CBD - rather than two variables - distance and direction - is critical in greatly simplifying the analysis of otherwise extremely cumbersome analytical models of urban structure. Moreover, as pointed out by Mills and Hamilton [1994, p. 127], in spite of this complexity, models which introduce two-dimensional description of location yield only few analytical results.

Notwithstanding the theoretical appeal of the strong uniformity and malleability assumptions which have been instrumental in sharpening our analytical understanding of the major forces which operate and interact with each other to shape urban structures, throughout the years an increasing number of scholars began to raise serious questions on the empirical validity of these assumptions. These studies are clear in showing that failure of each assumption to hold true will produce distorted estimates of density functions, thus invalidating the standard approach.

To remedy the situation three major lines of investigations distracting from these assumptions were pursued in the body of literature that emerged. One line of investigation posits that divergent from ideal spatial uniformity emerges due to the polycentric nature of many of today's large urban areas. Scholars such as Green [1980], Griffith [1981], Gordon et al. [1986], Small and Song [1993], McDonald and Prather [1993] and Alperovich and Deutsch [1996] conducted empirical investigations of structures of various metropolitan areas and reached the conclusion that thorough analysis of many large urban areas can by no means ignore their polycentric nature. Unfortunately while polycentric models are undoubtedly promising, nevertheless they leave a lot to be desired both theoretically and empirically, as the approach is not yet fully developed. 
A second line of investigation addresses the possibility those assuming monocentric urban areas irregular non-smooth patterns may still emerge at different distances from the CBD. This line of investigation was initiated by Anderson [1982, 1985]. The basic justification provided for the approach is that as dynamic forces operate to reshape urban structures over time and density gradients are becoming flatter it is unlikely that these forces will follow any structured distance variable. Consequently, we can expect emergence of irregular patterns with breaks in the smooth patterns of population density derived with the theoretical models. In this case Anderson [1982, 1985] suggests that an appropriate way to estimate population density functions allowing for such distance- oriented irregularities is to use a generalized cubic-spline specification. This function is cubic between "knots" of the function, is continuous in the first and second derivatives at the knots, but is discontinuous in the third derivative at the knots. The approach was subsequently adopted also to four more US urban areas by Mahmassani et al. [1986] and by Alperovich [1995] to the Tel-Aviv area. A major limitation of utilization of the cubic-spline specification uncovered in Alperovich [1995] is that due to multicollinearity in distance variables in many cases it is unsuitable for hypotheses testing.

A somewhat different approach along the same line of reasoning was taken by Anas [1978], Brueckner [1986] and Weathon [1982] among others. These authors proposed models embodying the more realistic assumption that housing capital is durable rather than malleable. In particular the models emphasize the importance of the age of structures in determining a neighborhood's population density. Discontinuous age patterns are a typical outcome of the redevelopment process thus generating discontinuous density contours. In an important contribution Brueckner [1986] proposed the application of the switching regression model for estimating inherently discontinuous density functions. In his application Brueckner [1986] estimated distance- oriented density functions allowing for an unknown number of possible regimes. The problem of choosing the number of different regimes is viewed as a problem in model selection. This is done using several generalized versions of the Information Criterion for model selection introduced by Akaike [1973].

In this paper we focus attention on the treatment of yet another possible type of spatial irregularities previously ignored by the literature. Specifically, in many cases we find that many irregularities described in the literature are inherently localized in nature and need not be distance- oriented. Irregularities of this type may arise due to topographical differences; differences associated with transportation systems and accessibility, differences associated with 
certain areas being socially less attractive than others or differences associated with redevelopment programs that are typically carried-out independent of distance. Such irregularities are best described by employing a directional variable. Spatial irregularities in urban structure of this type can duly be investigated empirically by using an extended model in which population density at each location is represented both by distance and direction. Utilizing a two-dimensional variate in this particular case simplifies the analysis by permitting the identification and estimation of density functions when an urban area is characterized by more than one regime.

The purpose of this paper is to propose and highlight a statistical estimation procedure designed for joint identification of irregularities in population distribution in urban areas which have a directional dimension and estimation the parameters of the model's separate regimes. The method we propose here is an application of the switching regimes regression technique developed by Quandt [1958, 1960] for identifying the most likely allocation of $n$ observations into two separate regimes, each associated with a different mechanism which generates its own set of observations. A subsequent purpose is to apply empirically the switching regimes method to the city of Tel-Aviv - Yafo and discern possible regimes where population densities are generated by different processes.

As is evident in the preceding two paragraphs both our approach and Brueckner's approach utilize Quandt's switching regression method. However each approach is designed to address a different situation and may not be applicable for situations where the other is. In particular, Brueckner [1986] uses a one variable -distance- to describe population density in different locations. Thus, the approach is most relevant when population density is independent of the direction of the location relative to the CBD. In contrast, we use two variables - distance and angle direction from CBD - to describe the density at each location. Thus if discontinuity in density contours is due to redevelopment or other factors that are angular specific, as one can expect in many realistic situations, Brueckner's approach will not be applicable in identifying the regimes while ours will. However, if discontinuity has no angular dimension our approach may still be valid and theoretically can be used to detect distance oriented breaks.

In a paper on population distribution in Tel-Aviv - Yafo, Alperovich [1995] found that the traditional negative exponential function does not perform well in explaining population distribution in recent years. In contrast, when the city was divided ad hoc into two sub-sections 
the performance of the model has markedly improved. It is our view that these initial findings make the city of Tel-Aviv - Yafo an ideal candidate for application of the proposed method. Considering the different patterns of development of the rapidly developing northern and the stagnating southern parts of the city it is expected that our analysis will be successful in identifying these regimes and each regime discerned will include tracts only from one of the above sections of the city. Notwithstanding this earlier finding it is important to emphasize at this juncture that the estimation procedure proposed in this paper does not require or make use of any a priori information about such a break. Rather the break is endogenously determined by the procedure as is standard in switching regime regression.

\section{Analytical Framework}

In developing our approach to modeling urban population distribution under directional spatial irregularities let us start by accepting the classical analysis by Muth [1969] and Mills [1972]. Accordingly, in monocentric urban areas where non-residential economic activities are concentrated in the $\mathrm{CBD}$, land is uniform in all distances and directions and housing capital is malleable population distribution around the CBD will follow an exponential function:

$$
D_{i}=D_{0} e^{-\eta u+\varepsilon}
$$

where, $D_{i}$ is population density $u$ kilometers from the $\mathrm{CBD}, D_{0}$ is a parameter which describes the density near the CBD, $\gamma$ is the density gradient which describes how rapidly density falls off with distance and $\varepsilon$ is a random disturbance term having zero mean and constant variance $\sigma_{\varepsilon}^{2}$.

Suppose now that due to non-uniform spatial economic and non-economic conditions population densities within a given slice of the urban area with a particular angular direction diverts from the uniform pattern described by (1). Such a diversion may emerge due to several factors. For instance, land in a given direction may be significantly more attractive due to special topographical attributes deemed highly desirable by the economic units. A case in point is when we have an attractive lake viewed by households as most desirable residential neighborhood and consequently they are willing to pay premium rent for land in the vicinity of the lake. Likewise, certain locations may be economically more attractive than other locations due to their flexible transportation network which makes them highly accessible. Indeed, 
neighborhoods may also be less attractive if they are located on hilly land not suitable for residential construction.

In these and many other cases irregularities are, to a great degree, localized with an angular direction so that their attractiveness is not determined solely by their distance from the CBD. Irregularities originating from these sources can best be captured and described by using the polar coordinates $(u, \theta)$ of tracts around the CBD. Here $\theta$ equals the angular direction of the tract from the CBD measured in radians and $u$ equals its distance from the origin. Assuming that population distribution in each of two directional subsections of an urban area is determined by a separate process population density at any point around the CBD can duly be written as:

$$
D_{i}= \begin{cases}D_{01} e^{-\gamma_{1} u_{i}+\varepsilon_{l i}} & \text { when } \mathrm{i} \in \mathrm{S}_{1} \\ D_{02} e^{-\gamma_{2} u_{i}+\varepsilon_{2 i}} & \text { when } \mathrm{i} \in \mathrm{S}_{2}\end{cases}
$$

where, $i$ is an index of observations $(i=1 \ldots n), \varepsilon_{1 i}$ and $\varepsilon_{2 \mathrm{i}}$ are random variables assumed to be normally and independently distributed with means zero and variances $\sigma_{1}^{2}$ and $\sigma_{2}^{2}$ and $S_{1}$ and $S_{2}$ are two regimes within an urban area where population densities are generated by two different processes. This situation is illustrated in Figure 1, which displays a circular urban area with a $\mathrm{CBD}$ at point $\mathrm{O}$ and five localities represented by points $A_{j}(j=1 \ldots 5)$ whose distances from the center is given by the length of the dotted lines. The urban area is divided into 2 subsections $\mathrm{S}_{1}$ and $\mathrm{S}_{2}$, separated by the solid line $\overline{\mathrm{EOF}}$. Localities $A_{1}$ and $A_{2}$ are equally distant from the CBD, as depicted by the length of the dotted lines, but their corresponding population densities are different as each is located in a different regime in the urban section $S_{l}$ and $S_{2}$, respectively.

A few observations are noteworthy regarding the model embedded in (2). First, within the classical framework of Muth and Mills' model, (2) is a very general and flexible representation. Population densities are presumed to decline exponentially in all directions and differences between the two regimes are allowed to be exhibited in all parameters of the model. Second, equation (2) is indeed a generalization of the classical model (1) and reduces to (1) whenever we cannot accept the hypothesis that either $S_{1}$ or $S_{2}$ is an empty set. Finally, we do not require that the sizes of $S_{1}$ and $\mathrm{S}_{2}$, and for that matter the decomposition of tracts $i$ into two 
subsets $S_{1}$ and $S_{2}$ will be known a priori. Rather, we are looking for an estimation procedure amenable of determining endogenously the exact composition of each regime.

In general, assuming $n$ to be an even number of urban localities around the CBD, the total number of different ways to divide the urban area into two sections, each containing $k$ and $n-k$ localities, respectively, is given by $\sum_{k=3}^{n / 2}\left(\begin{array}{l}n \\ k\end{array}\right)$. The minimum theoretical partition for the model displayed in (2) must contain 3 localities since at least one degree of freedom is required to estimate the parameters $D_{0}$ and $\gamma$. Even with a relatively moderate number of localities such as 100 , the total number of partitions to be examined would require almost an infinite amount of computer time. Thus, our restriction that each regime will be composed only of adjacent localities alleviates this intractable problem. Specifically, assuming an urban area with a predetermined $\mathrm{CBD}$, the regimes to be delineated by our procedure will be defined by two directional radii extending from the CBD to the peripheries of the urban area. In order to apply our procedure to this situation the observations must first be ordered according to their angle. Such an ordering can duly be accomplished by using the polar transformation, with the CBD as origin, for setting the relative position of each tract according to its angle of the transformation.

Under the above assumptions, the log likelihood function of the model displayed in (2) is given by:

$$
\begin{gathered}
\ln L=-\frac{n}{2} \ln (2 \pi)-\frac{n_{1}}{2} \ln \sigma_{1}^{2}-\frac{n_{2}}{2} \ln \sigma_{2}^{2} \\
-\frac{1}{2 \sigma_{l}^{2}} \sum_{i \in S_{1}}\left(y_{i}-\alpha_{1}-\gamma_{1} u_{i}\right)^{2}-\frac{1}{2 \sigma_{2}^{2}} \sum_{i \in S_{2}}\left(y_{i}-\alpha_{2}-\gamma_{2} u_{i}\right)^{2}
\end{gathered}
$$

where $y_{i}=\ln D_{i}(i=1, \ldots n), \alpha_{1}=\ln D_{01}, \alpha_{2}=\ln D_{02}$ and $n_{1}, n_{2}$ are the number of tracts in each section such that $n_{1}+n_{2}=n$. Differentiating with respect to $\sigma_{1}^{2}$ and $\sigma_{2}^{2}$ and substituting the estimates in equation (3) we obtain the following reduced log likelihood function:

$$
\ln L=-\frac{n}{2} \ln (2 \pi)-\frac{n}{2}-\frac{n_{1}}{2} \ln \hat{\sigma}_{1}^{2}-\frac{n_{2}}{2} \ln \hat{\sigma}_{2}^{2}
$$

where, 


$$
\begin{gathered}
\hat{\sigma}_{l}^{2}=\sum_{i \in S_{l}}\left(y_{i}-\hat{\alpha}_{1}-\hat{\gamma}_{1} u_{i}\right)^{2} / n_{1} \\
\hat{\sigma}_{2}^{2}=\sum_{i \in S_{2}}\left(y_{i}-\hat{\alpha}_{2}-\hat{\gamma}_{2} u_{i}\right)^{2} / n_{2} .
\end{gathered}
$$

Maximum likelihood estimates of $\alpha_{j}, \gamma_{j}, \sigma_{j}^{2}(j=1,2)$ can readily be obtained from the OLS regressions estimated using the specific partition of the urban area into $S_{1}$ and $S_{2}$ which maximizes the reduced log likelihood function (4). This method is an extension of the pioneering work of Quandt [1958] on switching regimes, except that whereas his partition refers to time series data ours refers to space. This difference creates a further complication. In the Quandt case, the problem is to divide a time series of $n$ observations into two groups, one containing $1,2, \ldots, k$ observations and the other containing the remaining $(k+1),(k+2), \ldots, n$ observations. Since the estimation requires at least one degree of freedom, $k$ can range from a low $k=2$ to a high $k=n-3$, implying the calculation of $n-5$ pairs of regressions. When the partition axis is time, the first and last observations are atypical and cannot appear in the same group. In the present application where the partition axis is a two-dimensional variate and the urban area is circular around the CBD, one cannot preclude the possibility that the "first" and "last" observations can indeed be any two adjacent localities that could even be included in the same group. Therefore, to find the optimal partition in a spatial context, we must repeat the search procedure sequentially assuming alternately a different urban locality as our starting point.

To test the null hypothesis that no switch occurred in our sample against the alternative that one switch occurred the appropriate likelihood ratio statistic is:

$$
\lambda=\frac{\left(\hat{\sigma}_{1}^{2}\right)^{\frac{n_{1}}{2}}\left(\hat{\sigma}_{2}^{2}\right)^{\frac{n_{2}}{2}}}{\left(\hat{\sigma}^{2}\right)^{\frac{n}{2}}}
$$

where $\hat{\sigma}_{1}^{2}$ and $\hat{\sigma}_{2}^{2}$ are defined in equations (5) and (6) and $\hat{\sigma}^{2}$ is the mean residual sum of squares derived from a single regression applied to the entire sample. 
Unfortunately, it was observed by Quandt [1960] that the distribution of $-2 \ln \lambda$ is poorly approximated by a $\chi^{2}$ variable. Feder [1975], Worsley [1979] and Deutsch [1992] have analyzed the distribution of $-2 \ln \lambda$ for the time series problem. Unfortunately this distribution is not applicable for the present two- dimensional partition case. Fortunately, testing for uniformity between the two regimes in a spatial context can still be accomplished by deriving an empirical distribution for Quandt's statistic based on 5,000 random samples and comparing the actual likelihood ratio statistic obtained from (7) with the $95^{\text {th }}$ percentile of the derived empirical distribution. Under the null hypothesis that the observations were generated by a single regime, each of the 5,000 random samples was obtained from:

$$
\ln D_{i}=\alpha+\gamma u_{i}+\varepsilon_{i} \quad ; i=1, \ldots, 48
$$

where $\varepsilon$ is a random variable generated from a normal distribution with zero mean and unit variance, $\alpha=0, \gamma=1$ and $u$ is distance of 48 tracts delineated by the Center for Economic and Social Research of the Tel-Aviv - Yafo Municipality from the CBD. It is noteworthy that the

particular values assumed for $\alpha, \gamma$ and $\sigma_{\varepsilon}^{2}$ do not affect the results since, as shown by Quandt, the likelihood ratio is independent of these parameters. Finally, for each sample the value of $\lambda$ was calculated and the distribution of $-2 \ln \lambda$ was constructed based on the 5,000 results. Figure 2 displays the empirical distribution derived using this procedure. Table 1 displays some descriptive statistics and the $95^{\text {th }}$ and $99^{\text {th }}$ percentiles of the distribution.

\section{Table 1}

Descriptive statistics of Quandt's Statistic based on 5,000 random samples.

\begin{tabular}{ccccccc}
\hline Min & Max & S.D. & Mean & Median & P95 $_{95}$ & P 99 \\
\hline 10.87 & 96.41 & 7.76 & 28.54 & 27.25 & 42.88 & 52.79
\end{tabular}




\section{Empirical Analysis}

The estimation procedure outlined in the preceding section was applied to 48 out of 55 tracts delineated by the Center for Economic and Social Research of the Tel-Aviv - Yafo Municipality for the year 1979. The seven tracts not included in the analysis consist of tracts, which are overwhelmingly occupied by non- residential activities. In line with the theoretical model, which refers only to residential densities these tracts, cannot be part of the model and should not be included. The data needed for an application of the model displayed in equations (4), (5) and (6) are minimal and are limited only to population density in each tract and the coordinates of the tracts. Data on population density for each observation was gathered from Physical, Demographic and Socio-Economic Characteristics of City Neighborhoods of the TelAviv - Yafo Municipality [1982]. The coordinates of these tracts were furnished by the Center for Economic and Social Research of the Tel-Aviv - Yafo Municipality and as such are highly reliable. These coordinates were utilized to compute the polar transformation of each tract, with the CBD serving as the origin. The relative position of each tract was then arranged according to its angle of transformation.

Before we turn our attention to the application of the proposed method for joint identification of different regimes and estimation of their associated density functions it is instructive to use the entire observations for estimating the standard model (1) and evaluating its performance. The results of this regression, along with the associated $t$ values of the estimatesgiven in parentheses- are displayed in (9):

$$
\begin{gathered}
\ln D=2.274-0.082 u \\
(12.426) \quad(1.990) \\
F=3.959 \quad \bar{R}^{2}=0.059 \quad \text { S.E } E=0.710 \quad n=48 .
\end{gathered}
$$

As expected, the regression yields that the sign of the distance coefficient is negative and all the coefficients of (9) are significant. These results notwithstanding, a major problem overshadowing the above regression is that its $\bar{R}^{2}$ is remarkably low, revealing that this model is inadequate in explaining the variability of population densities in Tel-Aviv - Yafo in 1979.

To fully appreciate the implication of the latter finding it should be addressed with a view to results obtained in previous studies on temporal changes in population densities in Tel-Aviv - 
Yafo during 1961 through 1980. In two studies on this topic Alperovich [1980a, 1980b] found that the explanatory power of the standard negative exponential function persistently diminished through time to a point where serious questions can be raised as to the validity of the conventional model. Attempting to ameliorate the situation, different spline functions were recently employed in Alperovich [1995] with the aim to uncover the factors responsible for this deterioration and improve the performance of the model. Unfortunately, none of the alternative spline specifications employed provided a satisfactory explanation for the behavior of population densities in Tel-Aviv - Yafo in 1979. This unsettling result was reversed when extraneous ad hoc information was used to separate observations into two groups, one containing mainly observations from the northern part of the city and the other from the southern part.

It is against this background that the poor performance of (9) should be evaluated. This negative result, however, is consistent with development trends in Tel-Aviv - Yafo. Specifically, even a rudimentary scrutiny of development trends in land use reveals that the socalled northern section of the city has developed rapidly during the past two decades whereas the southern section, consisting mainly of the older part of the city and many blighted areas, remains stagnant. In different time periods neighborhoods within the southern section were chosen as targets for various urban renewal projects. Nowadays it is common knowledge that tracts in the northern part are exceedingly attractive residential locations whereas tracts in the southern section are not. This, of course, is reflected in the relative price of housing which is considerably more expensive in the northern part.

In this paper we propose that this situation is ideal for an application of our model and assessment of its merits. Specifically, in view of the preceding discussion it is expected that applying the model to data from Tel-Aviv - Yafo, will yield two regimes each containing tracts only from one section. Furthermore, tracts from the northern and southern parts will not be mixed together in any regime.

It is noteworthy that the estimation of two different population density functions for different sections of an urban area is based on the assumption of angular non-symmetry of the population density function around the CBD. Under the null hypothesis of rotationalsymmetry, regressing population density on the angular part of the polar coordinates and the distance from the CBD should yield that the coefficient of the angular variable is zero. 
Therefore, estimating such regression provides a direct test of the null hypothesis of rotational- symmetry around the CBD. To assure that group assignment is a random process, a confidence interval for the coefficient of the angular variable is constructed using bootstrap techniques, a statistical method proposed and developed by Efron (1979), and Efron and Gong (1983). Given the population densities of the 48 tracts, a random sample of 48 tracts are drawn with replacement and the coefficient of the angular variable is estimated by regressing population density on the angular part of the polar coordinates and on the distance of tracts from the CBD. Repeating this procedure 5,000 times, we derived the distribution of the regression coefficients and calculate a 95\% confidence interval. The results obtained employing this procedure are displayed in Table 2. It can readily be verified that the results reject the null hypothesis of rotational symmetry around the CBD (i.e., the coefficient of the angular variable is zero) at the $5 \%$ significant level as the confidence interval includes only negative values for the estimated coefficient.

Table 2

Bootstrap results for the regression coefficients based on 5,000 random samples

Confidence Intervals

\begin{tabular}{lrrrr} 
Variable & $5 \%$ & $95 \%$ & $1 \%$ & $99 \%$ \\
\hline angular & -0.1581 & -0.0089 & -0.2035 & 0.0478 \\
distance & -0.1785 & -0.0371 & -0.2122 & -0.0049
\end{tabular}

To avoid a situation whereby the number of tracts included in any regime is too low to permit a satisfactory estimation of population density functions the model was applied under the restriction that the number of observations in each regime will not be smaller than 15 . The results of this estimation are illuminating and provide strong confirmation for our general expectations on the separate processes, which operate in two discerned sections of the city. In general, the estimation procedure yielded two regimes, and with only one exception, each 
containing tracts only from one section of the city. Specifically, the northern regime contains 20 tracts, all of which are located north of the CBD. The southern regime contains the remaining 28 tracts including the CBD. Except for one tract - number 12 - none of the tracts in the southern regime are located north of the CBD. The latter result is indeed remarkable as this odd neighborhood (Neve Shareth) appears to be an exception among the northern tracts. In a study conducted by the Center for Economic and Social Research of the Tel-Aviv - Yafo Municipality [1978] which aimed at uncovering residents' attitudes towards a wide range of living conditions in the various tracts it was found that the willingness of residents to continue to reside in their present tract was considerably lower in tract 12 than it was in any other tract in the northern section. Only in seven other tracts, all of which are in the southern section, residents expressed stronger aversion for remaining in their present neighborhoods. In this sense, this tract is idiosyncratic which by no means can be claimed to belong to the northern section. It is, of course, reassuring that the switching regime method is potent enough to uncover such oddity, a finding that increases our confidence in the adequacy of the proposed method. To give the reader a visual feeling for Tel-Aviv area, the 48 census tracts and the regimes delineated, a map of Tel-Aviv along with the census tracts and regimes is presented in Figure 3. The CBD is located in tract 24 and the two regimes discerned are separated by the line $\overline{\mathrm{EF}}$. The odd tract "Neve Sharet" is tract 12.

The maximum likelihood estimates of the density functions for the northern and southern regimes delineated by our procedure, along with the associated t values, given in parentheses, are displayed in equations (10) and (11), respectively:

$$
\begin{gathered}
\ln D=3.242-0.233 u \\
(11.524)(4.853) \\
F=23.549 ; \bar{R}^{2}=0.543 ; n_{1}=20 ;-2 \ln \lambda=45 \\
\ln D=1.922+0.005 u \\
(5.388)(0.065) \\
F=0.004 ; \bar{R}^{2}=0.0002 ; n_{2}=28 .
\end{gathered}
$$


Even a rudimentary scrutiny of these results sheds light on the obscure picture of the urban structure, which emerged from the results of the standard model displayed in (9). Failure of the standard exponential function to perform well in explaining the variability of population densities is divulged to be associated with our failure to account for existence of two different regimes. Whereas the structure of population distribution exhibited in the northern regime conforms exceedingly well to the conventional model the structure uncovered in the southern regime is utterly incompatible with this model. Specifically, we see that the coefficient of distance is negative and highly significant for the northern regime but is positive and insignificant for the southern regime. Indeed, the value of Quandt's statistic $-2 \ln \lambda$ equals 45 and is greater that the $95^{\text {th }}$ percentile of the distribution displayed in Table 1, thus implying that the null hypothesis that the observations where generated by a single equation is rejected at the $5 \%$ level of significance.

Moreover, the explanatory power of the model measured by its $\bar{R}^{2}$ is highly respectable for the northern regime (0.543) and disappointingly low (0.0002) for the southern regime. Also population densities decline 0.233 percent per unit of distance as we move north of the CBD and remain constant, exhibiting a slight tendency to rise, when we move south of it. Clearly, the stark difference in the behavior of population densities between the two regimes is the main factor uncovered to be responsible for the poor performance of the standard model (9). To give the reader a visual feeling for the different population density functions derived with (9), (10) and (11) they are plotted in Figure 4. These graphs show clearly that the correct density function which recognizes existence of two separate regimes gives a completely different picture of population distribution in Tel-Aviv - Yafo from than the one derived with the standard model which gives a distorted picture. Specifically, with the standard model population densities are underestimated in tracts close to the CBD and overestimated in tracts away from it in the northern section and vice versa in the southern section. As one can readily verify the distance where we have reversal in the direction of distortion is different for the two regimes. Whereas, reversal occurs 6.42 kilometers north of the CBD it occurs only 4.55 kilometers south of it. 


\section{Summary and Conclusions}

Through the years urban economists have become increasingly apprehensive about the empirical relevancy of smooth exponential population density functions derived from the standard model of urban structure under assumptions of monocentricity, uniformity and perfect capital malleability. To remedy the shortcomings of the classical approach two lines of investigations diverting from these assumptions were pursued in the literature. One approach focuses on irregularities stemming from the polycentric nature of large urban areas while the other accentuated distance oriented irregularities.

In this paper we address another possibility which focuses on spatial irregularities that have a directional dimension. Irregularities of this type may arise due to topographical differences, differences in transportation systems and differences associated with certain areas being socially less attractive than other areas. It is proposed that in these cases an investigation of urban structures can best be conducted by applying a switching regimes regression model to polar data consisting of a bivariate representing the angular direction and distance of census tracts from the CBD. This method is most suitable for joint estimation and identification of separate regimes within an urban area when each regime has a well-defined direction and population densities in each regime are determined by a different mechanism.

Applying this method to a cross-section data from Tel-Aviv - Yafo in 1979 yielded a few interesting results. First, the procedure was successful in identifying two regimes; one which includes, with only one exception, all tracts from the northern section and the other which includes all tracts from the southern section plus an odd tract. This finding is in full agreement with land use trends long observed to operate in Tel-Aviv - Yafo.

Second, the only exception for a complete north-south dichotomy is tract no. 12 . Whereas this tract is located in the northern section of Tel-Aviv it was assigned by our procedure to the southern section. Notwithstanding this result, there is a lot to be said in favor of such assignment, which is supported by several other indicators. Specifically, in an independent survey conducted by Tel-Aviv municipality households residing in this tract expressed strong aversion for residing there, typical only of inhabitants of a large number of southern tracts. In addition the survey is very clear in showing that socio-economic conditions in this particular tract are at odd with those prevailing in other northern tracts yet, they are in full agreement with 
those observed in southern tracts. It is thus reassuring that the switching regimes method was successful in allocating this tract to the southern regime.

Third, the estimated population density functions for the two regimes show that the northern regime of Tel-Aviv - Yafo is behaving according to the classical model while the southern regime does not. Quantitatively population density declines 0.233 percent per unit of distance in the northern regime and does not change, even exhibiting a slight tendency to increase with distance, in the southern regime. Finally, applying the conventional model using the entire set of observations from the two separate regimes yields distorted results as the explanatory power of the model is untenable low and the estimate of the joint gradient is exceedingly low. As one can readily see in Figure 4, the combined gradient which is -0.082 , underestimates the decline in densities in the northern part and overestimates it in the southern part.

\section{References}

H. Akaike, Information theory and the extension of the maximum likelihood principle, in $2 n d$ International Symposioum on Information Theory, (B. Petrov and F. Csaki, Eds.), Akailseoniani-Kiudo, Budapest (1973).

G. Alperovich, Determinants of population density gradient in Tel-Aviv metropolitan area, Urban Studies, 17, 185-192 (1980).

G. Alperovich, Neighborhood amenities and their impact on density gradients, The Annals of Regional Science, XIV, 51-64 (1980).

G. Alperovich, The effectiveness of spline urban density functions: An empirical investigation, Urban Studies, 32, 1537- 1548 (1995).

G. Alperovich and J. Deutsch, Urban structure with two coexisting and almost completely segregated populations: The case of east and west Jerusalem, Regional Science and Urban Economics, 26, 171-187 (1996).

A. Anas, Dynamics of urban residential growth, Journal of Urban Economics, 5, 66-87 (1978). 
J. Anderson, Estimating generalized urban density functions, Journal of Urban Economics, 18, $1-10(1985)$.

J. Anderson, Cubic spline urban density functions, Journal of Urban Economics, 12, 55-67 (1982).

J. K. Brueckner, A switching regression analysis of urban population densities, Journal of Urban Economics, 19, 174-189 (1986).

J. Deutsch, Linear regression under two separate regimes: An empirical distribution for Quandt's log likelihood ratio, Applied Economics, 24, 123-127, (1992).

B. Efron, Bootstrap Methods: Another Look at the Jackknife, Annals of Statistics, 7, 1-26 (1979).

B. Efron and G. Gong, A Leisure Look at the Bootstrap, the Jackknife, and Cross-validation, American Statistician, 36-48, February (1983).

P.I. Feder, The log likelihood ratio in segmented regressions, Annals of Statistics, 3, 84-97 (1975).

P. Gordon, H. Richardson, and H. Wong, The distribution of population and employment in a polycentric city: The case of Los Angeles, Environment and Planning A, 18, 161-173 (1986).

D. Green, Recent trends in urban spatial structure, Growth and Change, II, 29-40 (1980).

D. Griffith, Modelling urban population density in a multi-center city, Journal of Urban Economics, 9, 298-310 (1981).

H. Mahmassani, M. Baaj, and C. Tong, Characterization and evolution of spatial density patterns in urban areas, Department of Civil Engineering, University of Texas at Austin, (1986).

J.F. McDonald and P.J. Prather, Suburban employment centres: The case of Chicago, Urban Studies, 31, 201-218 (1994).

E.S. Mills, Studies in the Structure of the Urban Economy, Johns Hopkins University Press, Baltimore (1972).

E.S. Mills and B.W. Hamilton, Urban Economics, 5th edition, Harper Collins College Publishers, N.Y., N.Y. (1994). 
R. Muth, Cities and Housing, Chicago University Press, Chicago (1969).

R.E. Quandt, The estimation of the parameters of a linear regression system obeying two separate regimes, Journal of American Statistics Association, 53, 873-880 (1958).

R.E. Quandt, Test of the hypothesis that a linear regression obeys two separate regimes, Journal of American Statistics Association, 55, 324-330 (1960).

K. Small and S. Song, Population and employment densities: Structure and change, Working paper, University of California- Irvine and University of Nevada- Reno (1993).

Tel-Aviv - Yafo Municipality, Attitudes Towards Living Conditions in the Neighborhoods Among Inhabitants, Special Survey no. 51, Center for Economic and Social Research (1978).

Tel-Aviv - Yafo Municipality, Physical, Demographic and Socio-Economic Characteristics of City Neighborhoods, Center for Economic and Social Research (1982).

W.C. Wheaton, Urban residential growth under perfect foresight, Journal of Urban Economics, 12, 1-21 (1982).

K.J. Worsley, On the likelihood ratio test for a shift in location of normal populations, Journal of American Statistics Association, 74, 365-367 (1979). 
Figure 1

Urban Regimes

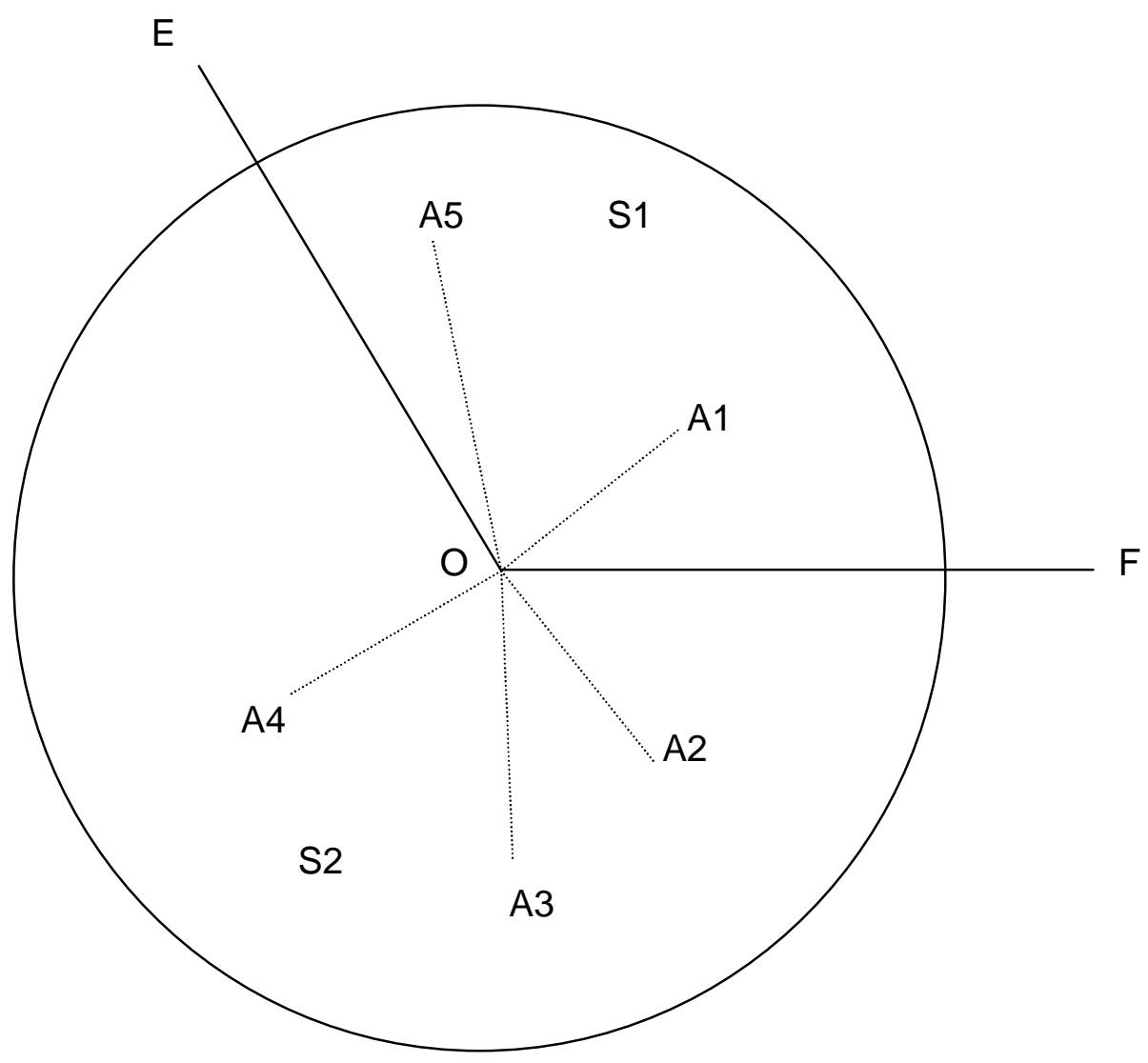


Figure 2

Empirical Distribution of $-2 \ln \lambda$

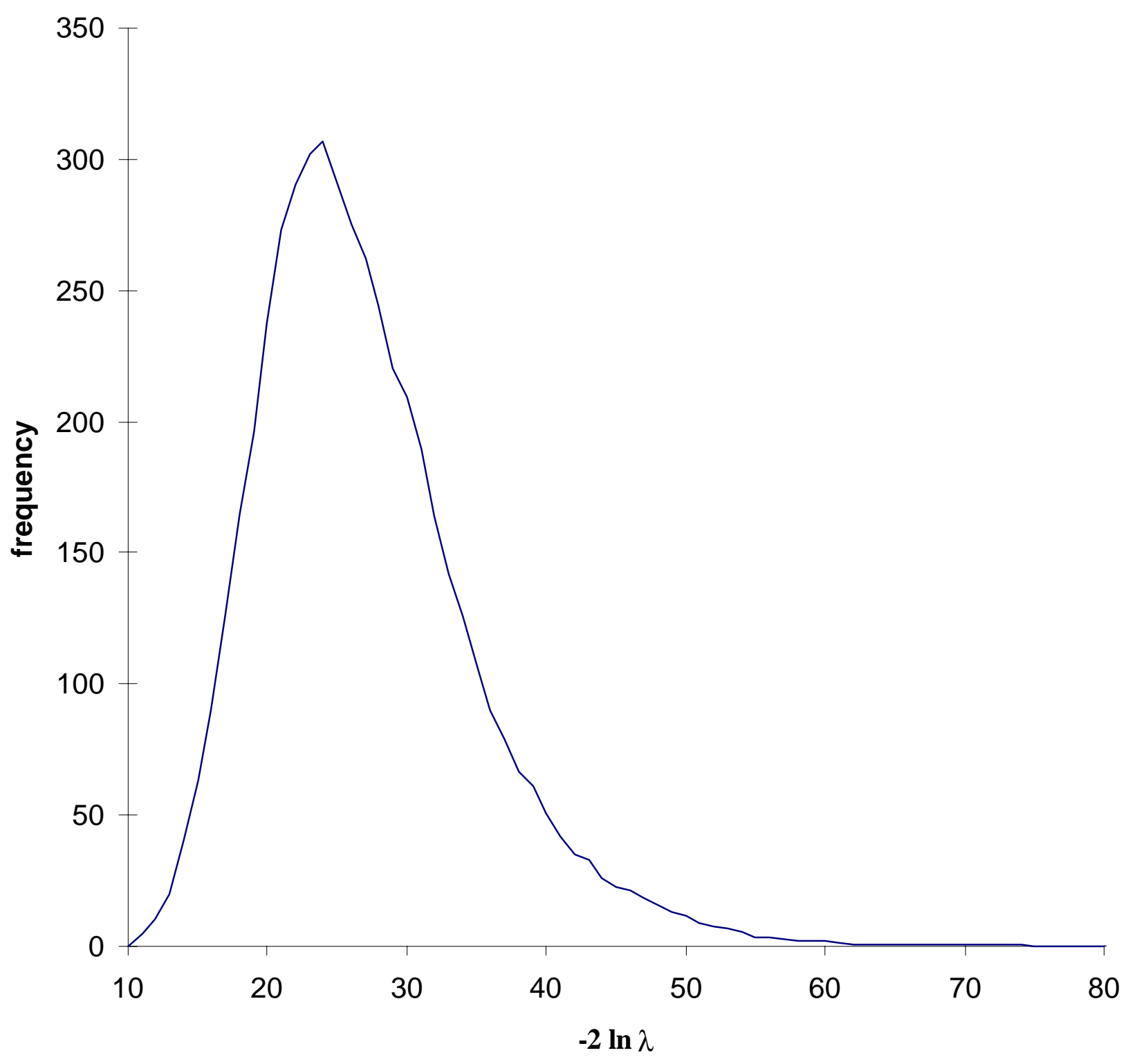


Figure 3

Tel-Aviv - Yafo Neighborhoods

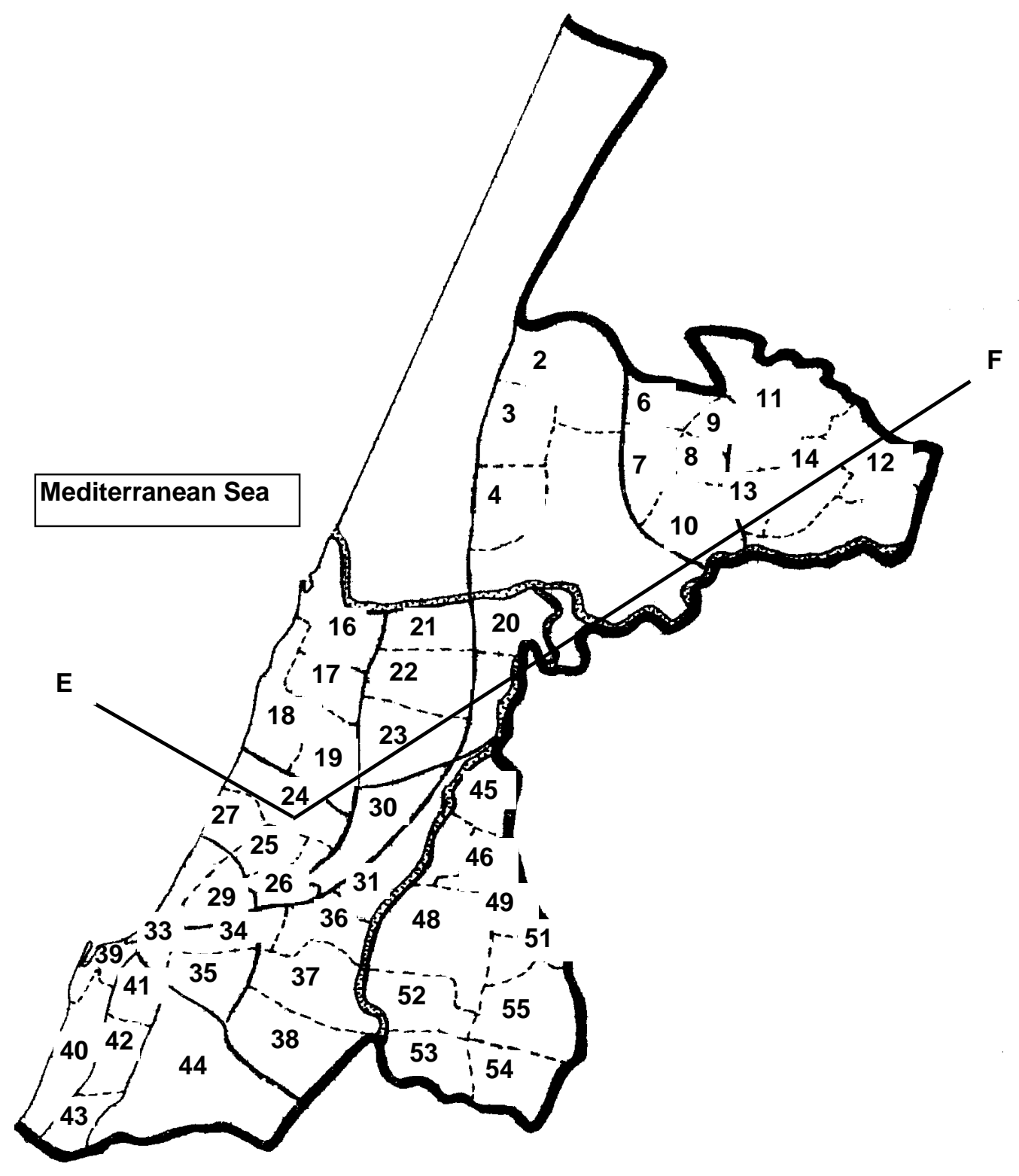


Figure 4

Density Functions for Tel-Aviv Yafo

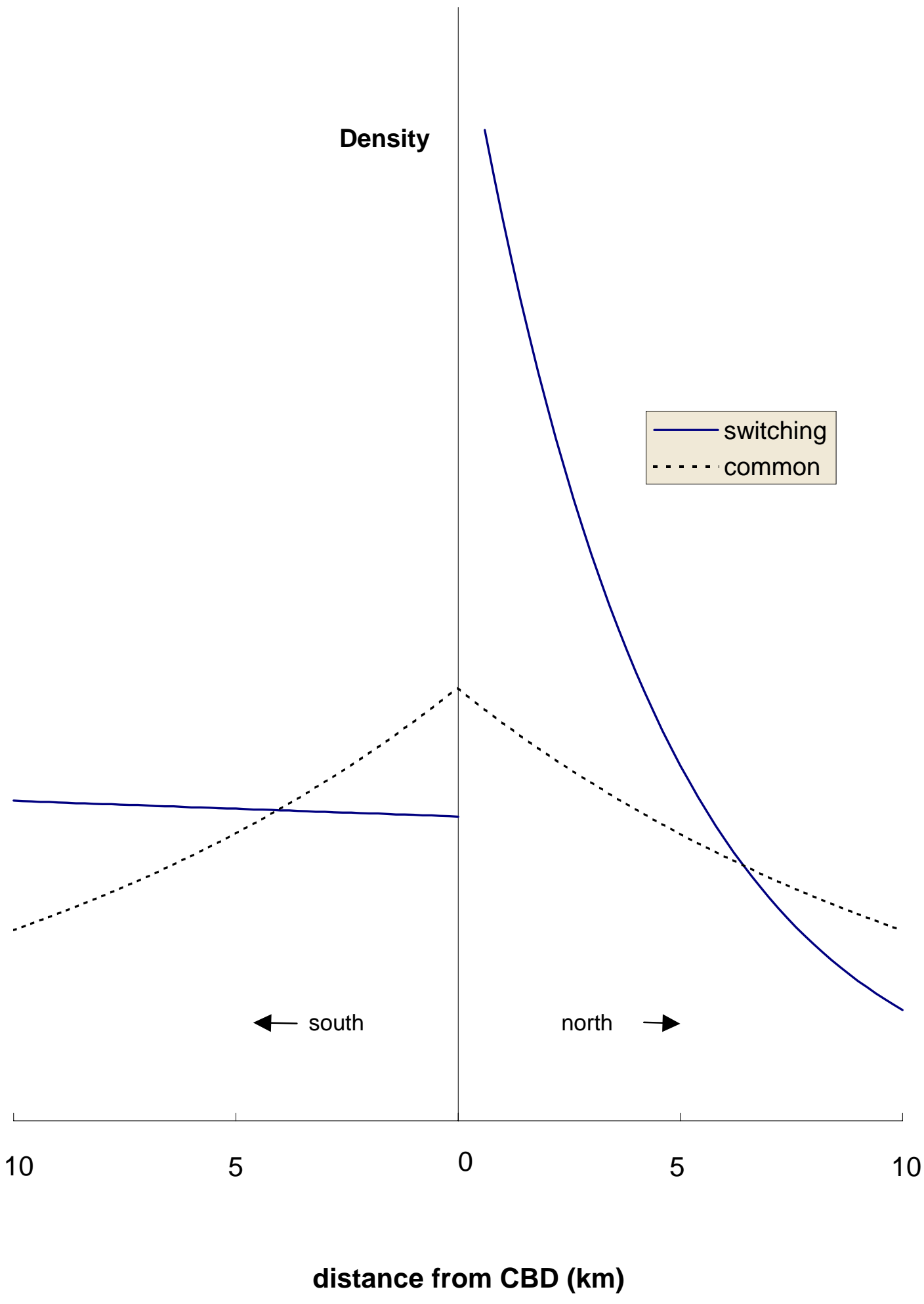

